\title{
Evaluation of ion-implanted-silicon detectors for use in intraoperative positron-sensitive probes
}

\author{
Raymond R. Raylman \\ Department of Internal Medicine, Division of Nuclear Medicine, University of Michigan Medical Center, \\ Ann Arbor, Michigan \\ Richard L. Wahl \\ Department of Internal Medicine, Division of Nuclear Medicine, and Department of Radiology, \\ University of Michigan Medical Center, Ann Arbor, Michigan
}

(Received 12 March 1996; accepted for publication 20 August 1996)

The continuing development of probes for use with beta (positron and electron) emitting radionuclides may result in more complete excision of tracer-avid tumors. Perhaps one of the most promising radiopharmaceuticals for this task is ${ }^{18} \mathrm{~F}$-labeled-Fluoro-2-Deoxy-D-Glucose (FDG). This positron-emitting agent has been demonstrated to be avidly and rapidly absorbed by many human cancers. We have investigated the use of ion-implanted-silicon detectors in intraoperative positronsensitive surgical probes for use with FDG. These detectors possess very high positron detection efficiency, while the efficiency for $511 \mathrm{keV}$ photon detection is low. The spatial resolution, as well as positron and annihilation photon detection sensitivity, of an ion-implanted-silicon detector used with ${ }^{18} \mathrm{~F}$ was measured at several energy thresholds. In addition, the ability of the device to detect the presence of relatively small amounts of FDG during surgery was evaluated by simulating a surgical field in which some tumor was left intact following lesion excision. The performance of the ion-implanted-silicon detector was compared to the operating characteristics of a positron-sensitive surgical probe which utilizes plastic scintillator. In all areas of performance the ion-implantedsilicon detector proved superior to the plastic scintillator-based probe. At an energy threshold of 14 $\mathrm{keV}$ positron sensitivity measured for the ion-implanted-silicon detector was $101.3 \mathrm{cps} / \mathrm{kBq}$, photon sensitivity was $7.4 \mathrm{cps} / \mathrm{kBq}$. In addition, spatial resolution was found to be relatively unaffected by the presence of distant sources of annihilation photon flux. Finally, the detector was demonstrated to be able to localize small amounts of FDG in a simulated tumor bed; indicating that this device has promise as a probe to aid in FDG-guided surgery. (c) 1996 American Association of Physicists in Medicine.

Key words: surgery, probe, semiconductor, positron

\section{INTRODUCTION}

Development of a small highly sensitive intraoperative probe for tumor localization has long been a goal of nuclear medicine. In the past several groups have utilized a wide variety of radiopharmaceuticals (most of which were labeled with gamma ray-emitting radionuclides) with appropriate detectors to localize focal regions of tracer uptake. ${ }^{1-16}$ Results from most of these studies revealed that two major drawbacks existed in this technique: (1) Tumor-to-background ratios were, for the most part, non-optimal for reliable differentiation of tumors, and (2) detection of distant sources of background gamma rays further reduced the already low tumor-to-background contrast. The discovery of the high affinity of ${ }^{18} \mathrm{~F}$-labeled-Fluoro-2-Deoxy-D-Glucose (FDG) in many human cancers ${ }^{17-20}$ inspired work on the use of this radiopharmaceutical for probe-guided surgery. Tumor-tobackground ratios averaging 8-to-1 have been reported by Wahl et al. ${ }^{21}$ In addition, to the excellent tumor contrast offered by FDG, the short range of the low energy positrons emitted by ${ }^{18} \mathrm{~F}(\sim 1.8 \mathrm{~mm}$ in soft tissue) facilitates precise tumor localization. While the limited range of particulate radiation was once viewed as a hindrance, this phenomenon is now seen as a potential advantage, since contamination of signal due to detection of non-tumor areas of positron emission is greatly reduced. There is, however, a major drawback in the utilization of a positron emitter; production of $511 \mathrm{keV}$ annihilation photons. Detection of these highly penetrating emissions greatly reduce the observed tumor-to-background contrast gained by the use of FDG. In response, detectors which are designed to maximize the detected positron-tophoton ratio have been introduced.

Most of the detectors proposed for use as positron probes have utilized plastic scintillators. ${ }^{22-27}$ Due to the low effective atomic number of this material, the detection for annihilation photons is small, while, given sufficient thickness, the positron detection efficiency approaches $100 \%$. Our group has demonstrated in preclinical animal trials the ability of a fiber-optic coupled plastic scintillator probe in tandem with FDG to guide the excision of human breast tumors. ${ }^{28}$ The device used in this study relied upon energy discrimination to reduce the photon contribution to the tumor signal. A probably more effective method for background photon rejection has been incorporated in a device invented by Daghighian et al $^{23-25}$ This probe consists of two concentric cylinders of plastic scintillator fiber-optically coupled to two photomultiplier tubes. The face of the outer hollow cylinder 
is covered by a $1 \mathrm{~mm}$ thick stainless steel sheet to prevent the passage of ${ }^{18} \mathrm{~F}$ positrons into the scintillator. The signals from this detector are used to correct for photon contamination of the signal from the inner scintillator which detects both photons and positrons. Correction of signal contamination is accomplished by a weighted subtraction of the outer detector count rate from the inner detector count rate. The weighting factor is the ratio of the gamma counting efficiencies of the two detectors, which is calculated during a relatively simple calibration procedure. The reported sensitivity of this probe to positrons emitted by ${ }^{18} \mathrm{~F}$ is $108 \mathrm{cps} / \mathrm{kBq} .{ }^{25}$

While the use of a second detector to measure the background contamination appears to be effective, this addition requires the probe tip to always be physically larger than just a single detector. Therefore, application of this type probe may be difficult where space is a premium, such as in intraluminal probes and situations where the surgical field is small. Smaller sized surgical areas are becoming more common as minimally invasive surgical procedures are developed. Furthermore, it is not clear that the background subtraction weighting function remains constant when the probe is presented with gamma rays which do not enter the detector volume through the front window; a situation which may be present in many common surgical applications. In order to develop probes which do not require background subtraction, so they can be miniaturized and do not necessitate a weighting function, we evaluated the use of semiconductor detectors.

The optimal positron-sensitive detector should possess close to $100 \%$ detection efficiency for positrons and a near $0 \%$ detection efficiency for $511 \mathrm{keV}$ annihilation photons. Most $\mathrm{Si}(\mathrm{Li})$ and $\mathrm{CdTe}$ detectors do have very nearly $100 \%$ positron detection efficiency, but because of their relatively thick depletion regions $(>1 \mathrm{~mm})$, detection efficiency for annihilation photons is undesirably high. Another class of semiconductor detectors, surface barrier detectors, possesses very near ideal characteristics. Indeed, Christian et al. have utilized surface barrier detectors to measure tracer kinetics in exposed canine hearts by detecting positrons emitted by ${ }^{62} \mathrm{Cu}-\mathrm{PTSM} .{ }^{29}$ While surface barrier detectors are good candidates for use as beta-sensitive intraoperative probes, another class of semiconductor detector closely related to these devices may be a better choice. Ion-implanted-silicon detectors (IISD) produce approximately $40 \%$ less noise than surface barrier detectors at body temperature ${ }^{30}$ and their operating parameters are more stable over a wider range of ambient conditions. In this investigation ion-implanted-silicon detectors are evaluated for future use as intraoperative positronsensitive probes. Since a separate detector to monitor background is not to be used, energy discrimination was employed to reduce background contamination of the positron signal.

\section{MATERIALS AND METHODS}

The ion-implanted-silicon detector used in this study was manufactured by EG\&G Ortec (Oak Ridge, TN). The Ul$\operatorname{tra}^{\mathrm{TM}}$ detector chosen possess a nominal $500 \mu \mathrm{m}$ thick deple- tion layer and was operated at a bias voltage of 100 volts. This amount of silicon is sufficient to stop most of the positrons emitted by ${ }^{18} \mathrm{~F}$. The factory specified beta energy resolution of this device is $4.2 \mathrm{keV}$. The circular silicon wafer which makes-up the sensitive area of the device has a diameter of $8 \mathrm{~mm}$. For the purposes of this initial investigation the detector unit was mounted in a light-tight aluminum cylinder. The entrance window consisted of a thin $\left(1.27 \times 10^{-3}\right.$ $\mathrm{cm}$ thick) sheet of aluminum foil. The cap on the other end of the cylinder contained the electrical feedthru required for biasing the device. Current pulses were conditioned with an EG\&G Ortec Model 142 charged-coupled preamplifier. The length of cable between the detector and preamplifier was minimized to help reduce noise and maintain good energy resolution. Amplification of the pre-amplified pulses was performed with an EG\&G Ortec Model 572 shaping amplifier (shaping time $=0.5 \mu \mathrm{s}$ ). The resulting voltage signals were routed to a pulse-height discriminator and the TTL pulses created by the discriminator were counted by a scaler module.

Many of the operating characteristics of the IISD where compared to those possessed by a plastic scintillation probe successfully tested in preclinical studies of FDG-guided breast cancer surgery. ${ }^{28}$ Briefly, this probe consists of a cylinder of BC-408 (Bicron Corp; Newbury, $\mathrm{OH}$ ) plastic scintillator (diameter $=8.4 \mathrm{~mm}$, length $=4 \mathrm{~mm}$ ) fiber-optically coupled to a photomultiplier tube (XP-1911; RCA Electronics). The plastic scintillator is enclosed in a stainless steel tube with a slanted front end piece to facilitate use in surgical procedures. This device employs energy discrimination as a means to reduce background photon detection.

Energy Spectra: The combined positron and annihilation photon energy spectrum measured by each detector was acquired by centering the detectors $0.4 \mathrm{~mm}$ above a circular $(8$ $\mathrm{mm}$ diameter) piece of filter paper containing $37 \mathrm{kBq}$ (1 $\mu \mathrm{Ci})$ of ${ }^{18} \mathrm{~F}$. Output from the amplifiers were "pulse-height analyzed" for 20 seconds by a Canberra Series 30 Multichannel Analyzer (Canberra Nuclear; Meriden, CT). A pure positron energy spectrum was obtained by covering the filter paper with a $0.2 \mathrm{~mm}$ thick piece of stainless steel, the Multichannel Analyzer was then set to subtract mode and a 20 second acquisition performed. The stainless steel will prevent positrons from reaching the detector. Thus the annihilation photon signal will be subtracted from the previously measured combined spectrum, resulting in a pure positron energy spectrum. This method will, however, cause a slight overestimation in the amount of photon background subtracted from the combined spectrum, since some of the annihilation photons produced in the stainless steel will not have been present in the original combined spectrum measurement. This error should be small, due to the relatively low detection efficiency for $511 \mathrm{keV}$ photons possessed by plastic scintillator and IISDs, and somewhat reduced by the slight attenuation of the photons by the thin plate of steel. Detected photon energy spectra were obtained by acquiring a spectrum for 20 seconds with the stainless steel in place.

Spatial Resolution: The spatial resolution of the IISD was 
measured by translating a $2.54 \times 10^{-2} \mathrm{~mm}$ thick piece of cotton thread soaked in ${ }^{18} \mathrm{~F}(15.5 \mathrm{kBq}[420 \mathrm{nCi}])$ across the face of the detector. The distance between the detector and thread was $0.4 \mathrm{~mm}$. At each point a series of 5,5 second acquisitions were obtained. A mean count rate and standard deviation for each position was calculated from this data. The resulting curve of count rate versus position was fit to a Normal distribution, and the full width at half maximum (FWHM) extracted as a measure of resolution. Measurements of resolution were acquired for a number of different threshold energies ranging from $6.8 \mathrm{keV}$ to $495 \mathrm{keV}$. To determine the effect of background radiation on the resolution of the IISD, a large circular petri dish containing 1.55 $\mathrm{MBq}(42 \mu \mathrm{Ci})$ of ${ }^{18} \mathrm{~F}(5.2 \mathrm{kBq} / \mathrm{ml}[0.14 \mu \mathrm{Ci} / \mathrm{ml}]$; this concentration simulates a standard uptake value (SUV) of 1 for an assumed injection of $370 \mathrm{MBq}[10 \mathrm{mCi}]$ to a $70 \mathrm{~kg}$ patient one hour prior to the surgical procedure) was placed behind the thread. The same method for acquiring and processing data to calculate resolution was performed.

Sensitivity and Selectivity: To measure detection sensitivity, a disk of filter paper ( $8 \mathrm{~mm}$ diameter) containing 55.5 $\mathrm{kBq}(1.5 \mu \mathrm{Ci})$ of ${ }^{18} \mathrm{~F}$ was positioned $0.4 \mathrm{~mm}$ below the front face of the IISD. A series of 5,5 second acquisitions were obtained from which mean and standard deviation counts per second were calculated. A second series of measurements, measuring only photon interactions, were obtained following the placement of a $0.2 \mathrm{~mm}$ thick piece of stainless steel in front of the detector face. By dividing the results from the first set of measurements by the amount of activity present on the disk, the combined (positron and photon) sensitivity was calculated. The positron sensitivity is calculated by first subtracting the pure photon count rate from the combined count rate and dividing by the amount of activity on the disk. Finally, the photon sensitivity was calculated by dividing the pure photon count rate by the amount of activity on the disk. The ability of the detector to distinguish between positron events and photon events, which we call selectivity, was determined by dividing the pure positron sensitivity by the photon sensitivity. All parameters were determined at energy thresholds ranging from $6.8 \mathrm{keV}$ to $495 \mathrm{keV}$. Both sensitivity and selectivity were determined for the IISD and plastic scintillation probe.

Residual Tumor Search: The most likely use of intraoperative probes will be to search for the presence of residual cancer left in the surgical field following attempts at tumor excision. To test the effectiveness of the IISD in this task, a surgical field following attempted lesion excision was simulated. Differing amounts of residual tumor were modeled by soaking various sized disks $(2 \mathrm{~mm}, 5 \mathrm{~mm}, 10 \mathrm{~mm}$ and 15 $\mathrm{mm}$ diameter) in a solution of ${ }^{18} \mathrm{~F}$ (concentration $=22.2$ $\mathrm{kBq} / \mathrm{ml}[0.6 \mu \mathrm{Ci} / \mathrm{ml}]$; simulating an SUV of 4.2 assuming a $370 \mathrm{MBq}[10 \mathrm{mCi}]$ infusion and a $70 \mathrm{~kg}$ patient). To model exposed normal tissue present in the tumor bed, a piece of filter paper $(7 \mathrm{~cm} \times 3 \mathrm{~cm})$ was soaked in a solution of ${ }^{18} \mathrm{~F}$ (concentration $=5.2 \mathrm{kBq} / \mathrm{ml}[0.14 \mu \mathrm{Ci} / \mathrm{ml}]$; simulating an SUV of 1 , assuming a $370 \mathrm{MBq}[10 \mathrm{mCi}]$ infusion and a 70 $\mathrm{kg}$ patient) and mounted on similar sized piece of tissue equivalent plastic (Gammex/RMI; Madison, WI). The tissue

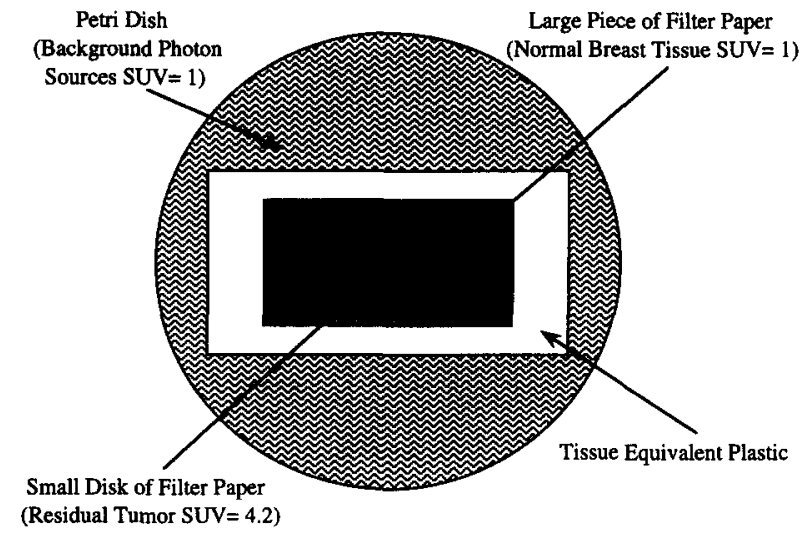

FIG. 1. Schematic drawing of a simulated surgical field following attempted excision of a lesion. Radiation signals from tumor remnants, tumor bed and internal sources are modeled.

equivalent plastic simulates tissue which contains little or no radionuclide located between major sources of FDG uptake (e.g. myocardium) and the tumor bed. This tissue will slightly attenuate the photon signal and completely absorb all emitted positrons. In order to simulate the background photon signal emanating from distant areas of normal tissue, a large petri dish (volume $=300 \mathrm{ml}$, diameter $=14 \mathrm{~cm}$ ) was filled with a solution of ${ }^{18} \mathrm{~F}$ (concentration $=5.2 \mathrm{kBq} / \mathrm{ml}[0.14$ $\mu \mathrm{Ci} / \mathrm{ml}]$; simulating an SUV of 1 assuming a $370 \mathrm{MBq}(10$ $\mathrm{mCi}$ ) infusion and a $70 \mathrm{~kg}$ patient). The large piece of filter paper and the piece of tissue equivalent plastic it was mounted on were placed on top of the petri dish. The disks simulating the tumor residue were then placed (one at a time) in the center of the large sheet of filter paper. Figure 1 shows a schematic of the final source configuration. A series of 5,5 second measurements were acquired while the probe was positioned $0.4 \mathrm{~mm}$ above the petri dish, the large piece of filter paper and the small disk of filter paper. Readings were acquired at a threshold energy of $14 \mathrm{keV}$. Mean and standard deviations calculated from this data were used to determine the Z-scores of the readings acquired above the large piece of filter paper (normal tissue) and the small disks (residual tumor), relative to the petri dish (sources of distant background) measurements. This experiment was repeated using the plastic scintillator probe. The SUVs used in this investigation represent the most commonly reported values. An SUV of 4.2 represents the low end of the values reported by Wahl $e t a l^{21}$ for breast tumors.

\section{RESULTS}

Energy Spectra: Figure 2 shows the ${ }^{18} \mathrm{~F}$ positron and 511 $\mathrm{keV}$ photon energy spectra obtained with the IISD and plastic scintillator detectors. Figure 2(a) also shows the theoretical shape of the positron energy spectrum calculated from the Fermi theory of beta decay. ${ }^{31}$ The two positron energy spectra obtained with the IISD presented in Fig. 2(a) illustrate that there is very little annihilation photon contamination of the combined spectrum. This is demonstrated by the very minor differences between the combined and positron 

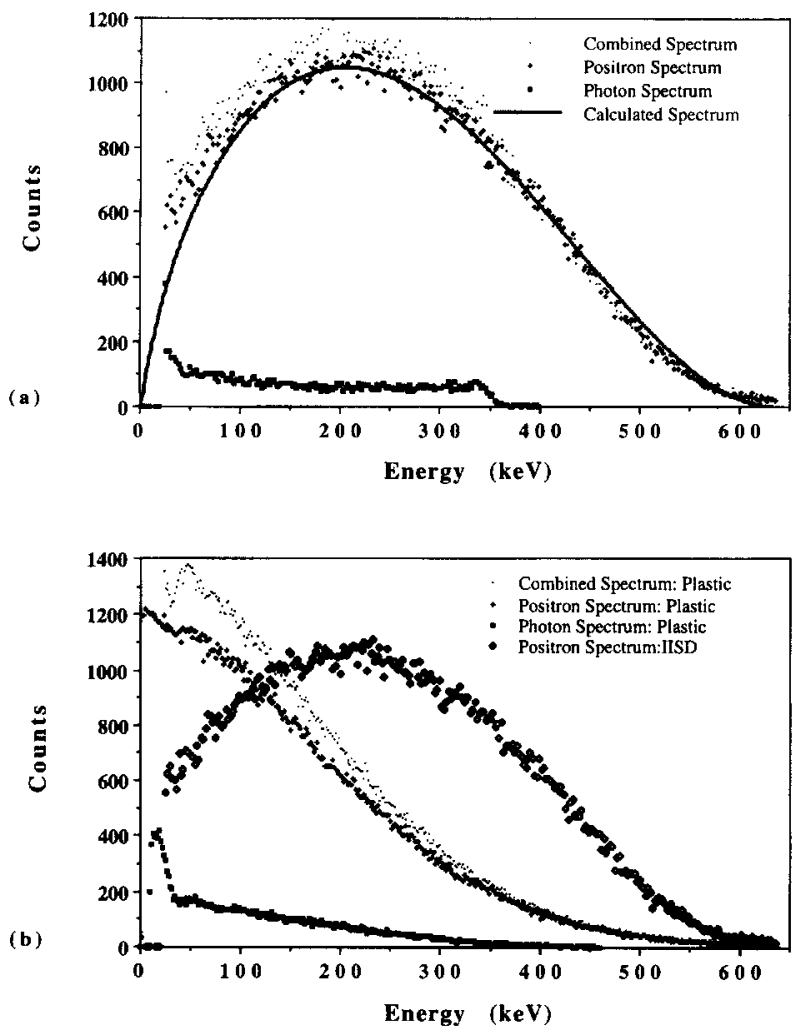

FIG. 2. Energy spectra measured using (a) the IISD, and (b) the plastic scintillator detector. Figure 2(a) also shows the predicted energy spectrum of positrons emitted by ${ }^{18} \mathrm{~F}$.

spectra. Also note that the minor differences in the spectra vanish at energies above the position of the Compton edge $(340.7 \mathrm{keV})$. In addition, the excellent energy resolution of the IISD is evident from the good agreement between the measured and calculated positron energy spectra. Figure 2(b) shows the energy spectra from the plastic scintillator detector in addition, for the sake of comparison, to the positron spectrum obtained with the IISD. Clearly, the positron energy spectra acquired with the plastic scintillator are distorted compared to the IISD spectrum. These spectra appear to have an over abundance of lower energy events.

Spatial Resolution: Figure 3 illustrates the change in IISD detector resolution as a function of energy threshold. Both curves, with and without the presence of background annihilation photon flux, are relatively flat; indicating a minimal dependence of resolution on threshold energy. In addition, the fact that the two curves are so similar supports the data displayed in Fig. 2(a) which shows that very little photon contamination of the positron signal is present.

Sensitivity and Selectivity: The plot in Fig. 4(a) compares the combined and positron sensitivities measured with the IISD as a function of threshold energy to the sensitivities measured with the plastic scintillator. Note that at most threshold energies the IISD has a higher positron detection sensitivity than the plastic scintillator. The graph in Fig. 4(b) demonstrates that the plastic scintillator has a higher photon detection sensitivity than the IISD at most threshold ener-

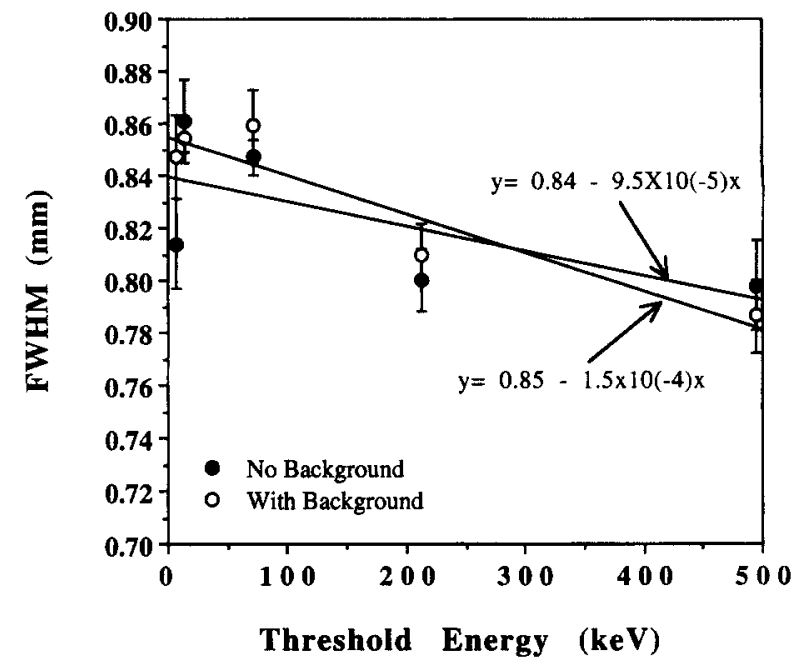

FIG. 3. Plot showing resolution of the IISD versus threshold energy setting. Also displayed are the fits to a straight line for the cases with and without the presence of background annihilation photon flux.

gies. The combination of a high positron detection sensitivity and low photon sensitivity results in a very high detected positron-to-photon ratio (selectivity) for the IISD, as demonstrated by the plot in Fig. 5 .
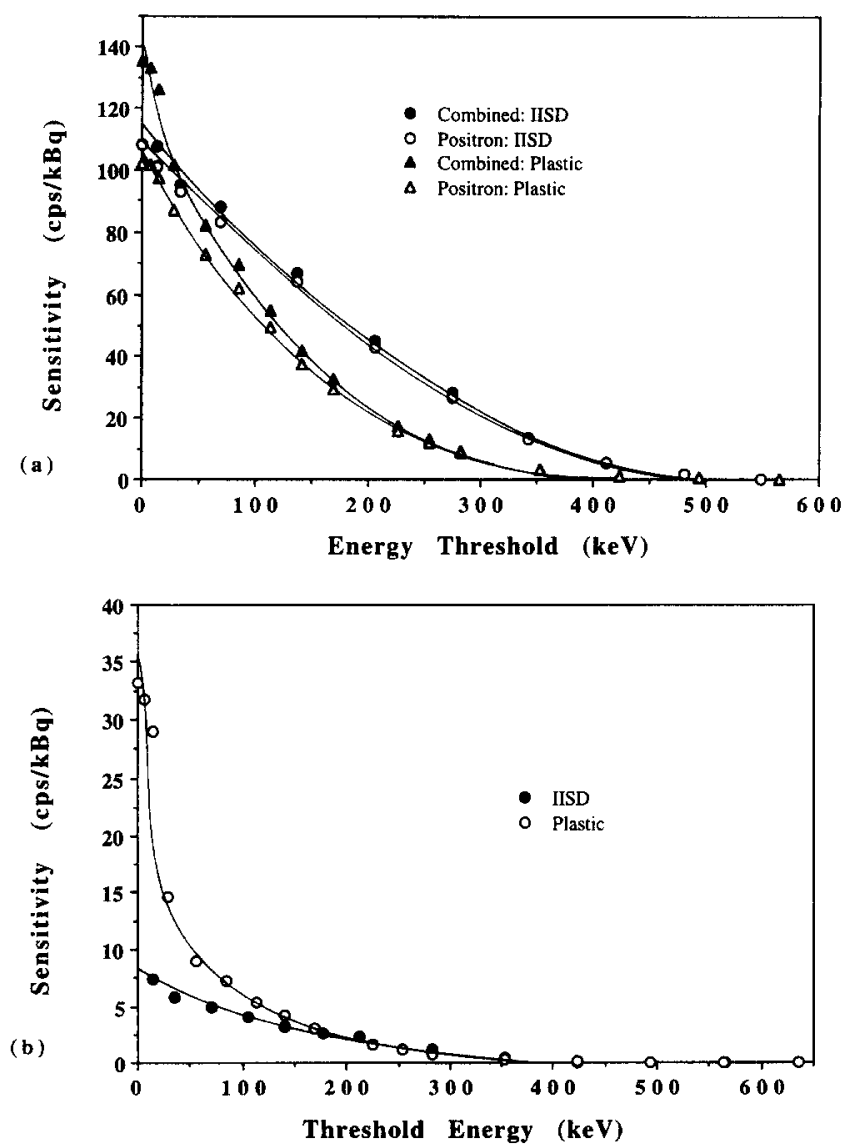

FIG. 4. The plot in (a) shows the detection sensitivity for positrons versus threshold energy setting, and (b) shows the detection sensitivity for $511 \mathrm{keV}$ photons versus threshold energy setting. Both plots display data acquired with the IISD and plastic scintillator. 


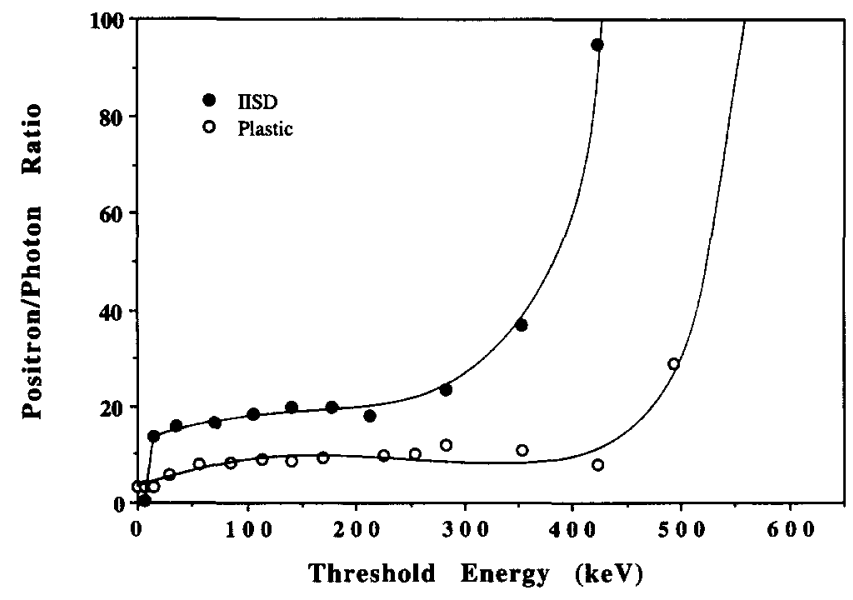

FIG. 5. Positron/photon detection ratio (selectivity) versus threshold energy.

Residual Tumor Search: The plot in Fig. 6(a) shows the results of the test to determine if it is possible to locate simulated residual FDG-avid disease in a tumor bed. Graphs of $Z$-scores versus disk size for the cases where the IISD detector was placed above the simulated tumor bed and above the simulated residual tumor are displayed. The plot in Fig. 6(b)

(a)

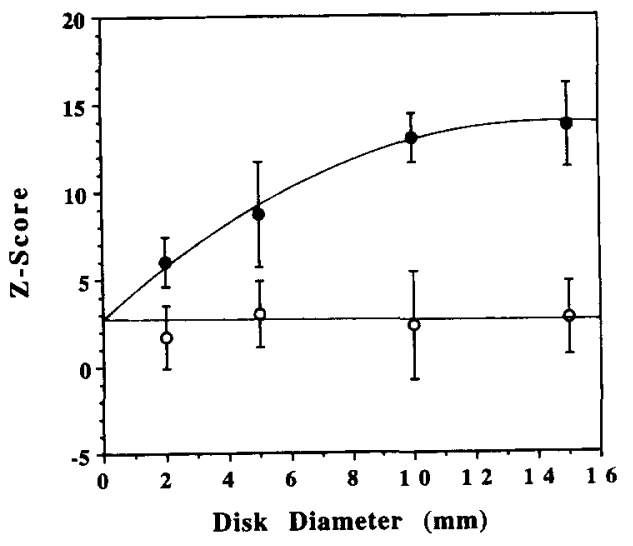

(b)

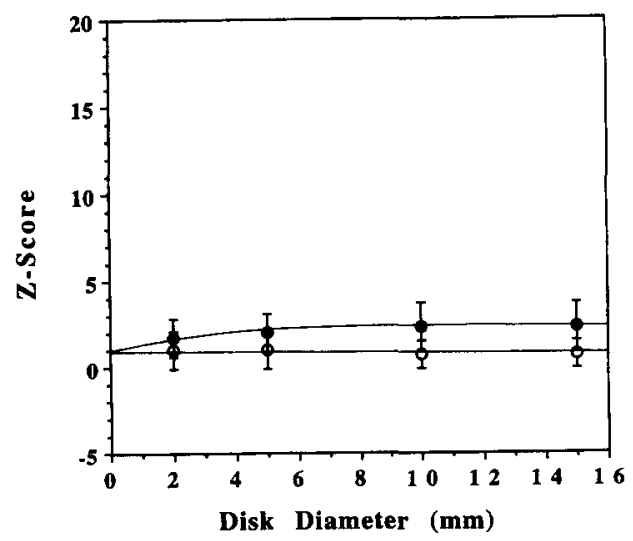

FIG. 6. Z-score versus disk diameter; z-score from measurements with the detector over the surrounding paper $(O)$ and the small disk (O). The plot in (a) shows results from measurements made with the IISD. The threshold energy setting was $14 \mathrm{keV}$. (b) shows the results for the search with a plastic scintillator probe. The energy threshold was $170 \mathrm{keV}$. shows the results for the plastic scintillator with the energy threshold set at $170 \mathrm{keV}$. At energy thresholds below 170 $\mathrm{keV}$ signal from the disks of activity were completely indistinguishable from background.

\section{DISCUSSION}

Since energy discrimination of the signals from the detector is to be used to reduce photon contamination, the positron and photon energy spectra measured by the detector are important. The spectra in Fig. 2(a) demonstrate the very good response of the ion-implanted-silicon detectors to positrons emitted by ${ }^{18} \mathrm{~F}$. It is important to note that measured positron energy spectrum agrees very well with the positron energy spectrum calculated for ${ }^{18} \mathrm{~F}$. Thus, it seems that very little energy is lost in the window of the detector. In addition, the position of the Compton edge measured by the IISD correlates reasonably well with the calculated value of $340.7 \mathrm{keV}$ for $511 \mathrm{keV}$ photons. Hence, it appears that, from examination of these spectra, the IISD and its associated electronics are producing the expected results.

While Fig. 2(a) demonstrates the very good performance characteristics of the IISD, the energy spectra in Fig. 2(b) illustrates that there are some problems with the plastic scintillator detector. The positron spectrum appears distorted and skewed towards the low energy end of the scale, compared to the IISD spectrum, thus indicating that not all of the scintillation light from each event is being collected. Little light should be lost in the transmission of the light pulses to the photomultiplier tube due to attenuation, since the attenuation of the fiber optic cable is small $(\sim 300 \mathrm{~dB} / \mathrm{km})$ and only $0.001 \mathrm{~km}$ of cable were used. Furthermore, since the light attenuation length of the scintillator is $210 \mathrm{~cm}$, very little self attenuation is occurring in the $4 \mathrm{~mm}$ long cylinder of $\mathrm{BC}$ 408. There are several possible sources of light loss in the system, however. First, some of the light pulses may undergo numerous reflections from the walls of the scintillator, and, although reflective coatings were applied, multiple reflections might produce some attenuation of signal. Also, there is a slight mismatch between the indices of refraction of the optical coupling compound and the scintillator. The index of refraction of the optical coupling compound is 1.46 and that of the scintillator is 1.58 . The critical angle for total internal reflection, therefore, is $67.5^{\circ}$. Hence, some of the light signals are not coupled to the fiber optic cable.

The effect of background photon contamination on IISD resolution appears to be minimal. The data presented in Fig. 3 indicates that spatial resolution as a function of energy threshold remains virtually unchanged; even when an annihilation photon background flux representative of that anticipated in a normal human upper chest is added. This is an improvement over some other plastic scintillation detectors which use energy discrimination to remove background contamination. ${ }^{27}$

Detection sensitivity is at least as important of an operating characteristic as is resolution. The data shown in Fig. 4(a) reveals the excellent sensitivity of the IISD detector. Indeed, at an energy threshold of $14 \mathrm{keV}$, combined detec- 
tion sensitivity is $108.7 \mathrm{cps} / \mathrm{kBq}$, which is virtually identical to that reported by Daghighian et al. $(108.0 \mathrm{cps} / \mathrm{kBq})$ for the dual plastic scintillation probe. ${ }^{25}$ At this threshold energy, the pure positron sensitivity is $101.3 \mathrm{cps} / \mathrm{kBq}$. As expected, detection sensitivity decreases rapidly as the energy threshold is increased. But, because of the skewed shape of the plastic scintillator positron energy spectrum, the reduction in sensitivity as a function of energy threshold is steeper than for the IISD. This effect is most prominent at low threshold energy values. Combined signal sensitivity is also greater than pure positron sensitivity due to detection of signal from annihilation photons. The difference between combined and positron sensitivity is most pronounced in the data from the plastic scintillator. The large disparity is due mostly to the higher photon detection cross section of this plastic scintillation detector compared to the IISD, as demonstrated by the data presented in Fig. 4(b). Sensitivity to photons can be reduced by using a thinner piece of plastic scintillator. For total absorption of all of the positron emitted by ${ }^{18} \mathrm{~F}$ the piece of scintillator must be at least $2 \mathrm{~mm}$ thick. One disadvantage of using a thin piece of scintillator is the potential of reduction in the light output per event. An additional advantage of using a thin detector, however, is a reduced cross section for detection of photons originating from areas located to the sides of the detector. The presence of sources of radiation which are not parallel to the face of the probe is common in situations where the device is used to survey a tumor bed. Proper shielding can eliminate the effect of positron flux. The photon flux, however, is very difficult to shield, so the geometry of the detector must be optimized to reduce detection. Since the IISD has a much thinner active area (500 $\mu \mathrm{m}$, in this case, or less) than most scintillation detectors, the detection of "off-axis" source of photons will be significantly reduced. Hence, IISDs (or other types of thin semiconductor detectors such as surface barrier detectors) will be less sensitive to detection of off-axis sources of photons which are almost certainly going to be present in surgical applications.

Perhaps the most important operating characteristic of a positron sensitive detector for use in FDG-guided procedures is the ratio of detected positron-to-photon events. Figure 5 shows the ratio between positron and photon sensitivity as a function of threshold energy. The differences in the data for the IISD and plastic scintillator at the lower and intermediate threshold energies are due to the higher photon detection cross section and lower positron sensitivity of the plastic scintillator detector at these energy threshold settings. At higher energies, the positron/photon ratio for the plastic detector doesn't increase at the same energy as the IISD. This phenomenon is due primarily to the poor energy resolution of the plastic scintillator.

The data presented in Fig. 5, in conjunction with the data displayed in Fig. 4, can be used to determine the optimal threshold energy setting for the IISD. Clearly, a trade-off in operating characteristics must be made. From Fig. 4 we see that the greatest sensitivity is achieved at very low threshold settings. This phenomenon is due to the detection of annihilation photons and noise present in the detector, in addition to the positron events. Therefore, the selectivity should be poor. This effect is confirmed by the data shown in Fig. 5 . Also we see from Fig. 5 that, because of the complete exclusion photon events, selectivity is greatest at threshold energies above $\sim 350 \mathrm{keV}$. Since ${ }^{18} \mathrm{~F}$ emits relatively few positrons with energies greater than $350 \mathrm{keV}$ [illustrated by the plots in Fig. 2(a)], sensitivity at these settings is poor. Therefore a compromise must be made. Because the positron/ photon ratio is relatively constant from a threshold energy of $14 \mathrm{keV}$ to $211 \mathrm{keV}$, the threshold setting which results in the greatest sensitivity was chosen, $14 \mathrm{keV}$.

Having decided upon the optimal energy threshold value for the IISD, the use of this detector in a simulated search for tumor remnants was performed. The data shown in Fig. 6(a) demonstrates the potential effectiveness of this device in this application. Even when the amount of ${ }^{18} \mathrm{~F}$ and the disk were smallest $(2 \mathrm{~mm}$ and $0.52 \mathrm{kBq}[14 \mathrm{nCi}]$, respectively), the $\mathrm{Z}$-score was greater than simulated surrounding tissues. As the disk and total amounts of activity grew, the $Z$-score relative to the simulated background activity grew, until the disk size exceeded the diameter of the detector $(8 \mathrm{~mm})$. At the point where the diameter of the disk was greater than the detector, those positrons and photons not emitted from the areas viewed by the IISD were not detected. Hence, the static $\mathrm{Z}$-score calculated for the $15 \mathrm{~mm}$ diameter disk. While the IISD was able to detect the disks of activity in the presence of sources of background, the plastic-scintillator probe had a great deal of difficulty in accomplishing this task. Indeed, in order to approach the performance of the IISD, the energy threshold had to be set to $170 \mathrm{keV}$. At this level the sensitivity to positrons was measured to be $29.4 \mathrm{cps} / \mathrm{kBq}$, in contrast the IISD's positron sensitivity at $14 \mathrm{keV}$ (the level utilized in the experiment) of $101.3 \mathrm{cps} / \mathrm{kBq}$. Clearly, more realistic tests utilizing much more complex source geometries and phantoms will be necessary to definitively identify the capabilities of an IISD-based surgical probe in actual use. These studies are now in progress.

The superiority of the IISD in detecting small areas of simulated FDG-avid tissue is very significant to the future application of this type of detector to FDG-guided surgery. These detectors perform better than a plastic scintillatorbased probe which uses energy discrimination, and should be smaller in size and possibly have superior performance compared to a dual probe design. An important advantage to IISDs may be that they can be made very small. Thus, facilitating their application in endoscopy, colonoscopy and laparoscopy, in addition to guidance of the complete excision of tumors. Since IISDs do not rely on the collection of scintillation light, the problems associated with reduced light output from the detector material, and the often bulky and complex systems to collect and process these optical signals is avoided. Furthermore, an intraoperative probe utilizing an IISD would be very simple to operate and should not require special calibration procedures or signal processing. Although this investigation utilized a positron-emitting radionuclide $\left({ }^{18} \mathrm{~F}\right)$, IISDs are capable of detecting positrons or electrons with comparable efficiency. Thus, IISD-based probes could be used with more common radiopharmaceuticals labeled 
with ${ }^{131} \mathrm{I},{ }^{32} \mathrm{P}$ and possibly ${ }^{111} \mathrm{In}$. While the preliminary results presented in this work are encouraging, future tests utilizing more complex phantom designs and tumor models in animals are necessary to define the role that ion-implantedsilicon detectors will play in radiopharmaceutical-guided surgeries.

${ }^{1}$ L. D. Marinelli and B. Goldschmidt, "The concentration of ${ }^{32} \mathrm{P}$ in some superficial tissues of living patients,"' Radiology 39, 454-463 (1942).

${ }^{2}$ B. V. A. Low-Beer, H. G. Bell, H. J. Mc Corkle, and R. S. Stone, "Measurement of radioactive phosphorus in breast tumors in situ; a possible diagnostic procedure," Radiology 47, 492-493 (1946).

${ }^{3}$ B. Selverstone, W. H. Sweet, and C. V. Robinson, "The clinical use of radioactive phosphorus in the surgery of brain tumors," Ann. Surg. 130, 643-651 (1949).

${ }^{4}$ C. C. Harris, R. R. Bigelow, J. E. Francis, G. G. Kelley, and P. R. Bell, “A CsI(TI)-crystal surgical scintillation probe," Nucleonics 14, 102-108 (1956).

${ }^{5}$ A. C. Morris, Jr., T. R. Barclay, R. Tanida, and J. V. Nemcek, "A miniaturized probe for the detecting of radioactivity at thyroid surgery," Phys. Med. Biol. 16, 397-404 (1971).

${ }^{6}$ W. C. Harvey and J. L. Lancaster, "Technical and clinical characteristics of a surgical biopsy probe," J. Nucl. Med. 22, 184-186 (1981).

${ }^{7}$ T. S. Hinckernell, H. B. Barber, H. H. Barrett, and J. M. Woolfenden, "“ Dual-detector for surgical tumor staging," J. Nucl. Med. 29, 1101-1106 (1988).

${ }^{8} \mathrm{~K}$. L. Swinth and J. H. Ewins, "Biomedical probe using a fiber-optic coupled scintillator," Med. Phys. 3, 109-112 (1976).

${ }^{9}$ J. M. Woolfenden, W. S. Nevin, H. B. Barber, and D. J. Donohue, "Lung cancer detection using a miniature sodium iodide detector and cobalt-57 bleomycin," Chest 85, 84-88 (1984).

${ }^{10}$ Y. Sasaki, H. N. Wagner, Jr., M. Iio, S. Murao, S. Takayanagi, and T. Kobayashi, "Catheter semiconductor and its application to clinical and experimental medicine," in Semiconductor Detectors in the Future of Nuclear Medicine, edited by P. B. Hoffer, R. N. Beck, and A. Gottschalk (The Society of Nuclear Medicine, New York, 1971), p. 144-156.

${ }^{11} R$. Sassa, M. lio, and T. Sugito, "The diagnosis of the gastric cancer by radionuclides and their labels, using catheter-type semiconductor radiation detector (CASRAD)," Gastro. Jap. 15, 369-373 (1980).

${ }^{12} \mathrm{P}$. Lommatzsch, "Clinical experience with the ${ }^{32} \mathrm{P}$ test and the semiconductor probe in diagnosis of intraocular tumors," Mod. Probl. Opthal. 18, 73-76 (1977).

${ }^{13}$ C. A. Nierodi, C. Mojzisisk, A. Sardi, P. Fereara, G. Hinkle, M. O. Thurston, and E. W. Martin, "The impact of radioimmunoguidedsurgery (RIGS) on surgical decision-making in colorectal cancer," Dis. Col. Rect. 32, 927-932 (1989).

${ }^{14}$ A. Sardi, M. Workman, C. Mojzisik, G. Hinkle, C. Nieroda, and E. W. Martin, "Intra-abdominal recurrence of colorectal cancer detected by radioimmunoguided surgery (RIGS system)," Arch. Surg. 124, 55-59 (1989).
${ }^{15}$ A. M. Cohen, E. W. Martin, I. Lavery, J. Daly, A. Sardi, D. Aitken, K. Bland, C. Mojzisik, and G. Hinkle, "Radioimmunoguided surgery using iodine 125 B72.3 in patients with colorectal cancer," Arch. Surg. 126, 349-352 (1991)

${ }^{16}$ J. C. Alex, D. L. Weaver, J. J. Fairbank, B. S. Rankin, and D. N. Krag, "Gamma-probe-guided lymph node localization in malignant melanoma," Surg. Oncol. 2, 303-308 (1993).

${ }^{17}$ R. L. Wahl, G. D. Hutchins, and D. J. Buchsbaum, "Fluorine-18-2deoxy-2- glucose uptake into human xenografts,"' Cancer 67, 1550-1554 (1991).

${ }^{18} \mathrm{~N}$. Y. Tse, C. K. Hoh, and R. A. Hawkins, "The application of positron emission tomography imaging with fluoro-deoxyglucose to the evaluation of breast disease," Ann. Surg. 216, 27-34 (1992).

${ }^{19}$ O. E. Niewig, E. E. Kim, and W-H. Wong, "Positron emission tomography with fluorine18-deoxyglucose in the detection and staging of breast cancer," Cancer 71, 3920-3925 (1993).

${ }^{20}$ L. P. Adler, J. J. Crowe, N. K. Al-Kaise, and J. L. Sunshine, "Evaluation of breast masses and axillary lymph nodes with [F-18] 2-deoxy-2-fluorod-glucose," Radiology 187, 743-750 (1993).

${ }^{21}$ R. L. Wahl, R. L. Cody, G. D. Hutchins, and E. E. Mudgett, "Primary and metastatic breast carcinoma: Initial clinical evaluation with PET with radiolabeled glucose analog 2-[F-18]-fluoro-2-dexoy-d-glucose," Radiology 179, 765-776 (1991).

${ }^{22}$ R. A. Lerch, H. D. Ambros, S. R. Bergmann, B. E. Sobel, and M. M. TerPogossian, "Kinetics of positron emitters in vivo characterized with a beta probe," Am J. Physiol. 242, H62-H67 (1982).

${ }^{23} \mathrm{U}$. S. Patent Number 5008546: "Intraoperative beta probe and method of using the same," J. C. Mazziotta, F. Daghighian, and E. J. Hoffman.

${ }^{24}$ F. Daghighian, K. Ikezake, K. J. A. Kairemo, J. C. Mazziotta, K. L. Black, S. Siegel, J. M. Guerrero, W. Digby, E. J. Hoffman, and M. E. Phelps, "Beta sensitive intraoperative probe," J. Nucl. Med. Suppl. 31, 759 (1990) abstract.

${ }^{25}$ F. Daghighian, E. J. Hoffman, P. Shenderov, B. Eshaghian, S. Siegel, and M. C. Phelps, "Intraoperative beta probe: A device for detecting tissue labeled with positron or electron emitting isotopes during surgery," Med. Phys. 21, 153-175 (1994).

${ }^{26}$ R. R. Raylman and R. L. Wahl, "A small fiber-optically coupled plastic scintillation positron probe," J. Nucl. Med. Suppl. 34, 113P (1993) abstract.

${ }^{27}$ R. R. Raylman and R. L. Wahl, "A fiber-optically coupled postironsensitive surgical probe," J. Nucl. Med. 35, 909-913 (1994).

${ }^{28}$ R. R. Raylman, S. J. Fisher, R. S. Brown, S. P. Ethier, and R. L. Wahl, "Fluorine-18-fluorodeoxyglucose-guided breast cancer surgery with a positron-sensitive probe: Validation in preclinical studies," J. Nucl. Med. 36, 1869-1874 (1995).

${ }^{29}$ B. T. Christian, R. J. Nickles, and C. K. Stone, "AA surface barrier $\beta$ detector for measuring tracer kinetics in canine myocardium," J. Nucl Med. Suppl. 34, 184P (1993) abstract.

${ }^{30} \mathrm{EG} \& \mathrm{G}$ Ortec, Modular Pulse-processing Electronics and Semiconductor Radiation Detectors (EG\&G Ortec, Oak Ridge, 1995), p. 400.

${ }^{31}$ R. D. Evans, The Atomic Nucleus, 3rd ed. (McGraw-Hill, New York, 1976), pp. 278-279. 